\title{
Shock Wave Driven by an Expanding System of Loops
}

\author{
N.-E. Raouafi ${ }^{1}$, S. Mancuso ${ }^{2}$, S. K. Solanki ${ }^{1}$, B. Inhester ${ }^{1}$, M. Mierla ${ }^{1}$ \\ G. Stenborg ${ }^{3}$, J. P. Delaboudinière ${ }^{4}$, and C. Benna ${ }^{2}$ \\ ${ }^{1}$ Max-Planck-Institut für Sonnensystemforschung, 37191 Katlenburg-Lindau, Germany; \\ ${ }^{2}$ OATo, Torino, Italy; ${ }^{3}$ GSFC/CUA, Greenbelt, USA; ${ }^{4}$ IAS, Orsay, France
}

\begin{abstract}
We report on a Coronal Mass Ejection (CME) observed on June 271999 by the UltraViolet Coronagraph Spectrometer (UVCS) telescope operating on board the SOHO spacecraft. The CME was also observed by LASCO (SOHO). Emission of hot material has been recorded by UVCS propagating in front of an opening system of loops generated by the CME. The evolution of the UVCS structure is highly correlated to the evolution of the opening loop. The data reveal excess broadening of the $\mathrm{O}$ vi doublet lines and an enhancement in the intensity of the Si XII $\lambda 520.66$ and $\lambda 499.37$ lines due to the motion of the expanding hot gas. The hot gas emission seems to be due to a shock wave propagating in front of a very fast gas bubble traveling along the opening loop system.
\end{abstract}

Keywords. plasmas, shock waves, Sun: coronal mass ejections (CMEs), UV radiation

\section{Introduction}

UVCS observations of CMEs usually show emission in low to moderate ionization stages. In a few cases UV spectra show emission from higher ionization states that can be interpreted in terms of coronal shock waves connected with the CME eruptions. The CME observed by UVCS on June 271999 produced a strong enhancement of the Si XII emission lines originating from hot plasma seen propagating along the slit.

\section{Observations and data analysis}

The results discussed in the present paper have been obtained from observations made on June 271999 by different instruments aboard the SOHO spacecraft. The analysis of this event also relies on radio spectra recorded by the decametric array of Nançay (France) and the Izmiran radio spectrograph (Russia). More details about the data analysis are given in Raouafi et al. (2004).

Three UVCS exposures taken at $2.55 R_{\odot}$ reveal the propagation of hot plasma with highly enhanced emission in the Si XII lines, which is rare in CMEs (see Fig. 1). These lines trace the propagation of hot plasma along the UVCS slit. A co-spatial broadening of the O VI doublet lines is also remarkable (the same figure).

The observed broadening of the two $\mathrm{O}$ vi emission lines, together with the mentioned enhanced Si XII emission and the simultaneous detection of a metric type II radio burst by ground radiospectrographs, is suggestive of the passage of a coronal shock wave (e.g. Raymond et al. 2000; Mancuso et al. 2002).

The CME was observed initially by EIT to erupt as a system of loops. The white light images (Fig. 2) show a structure propagating toward the north pole that has the same general characteristics of propagation as the Si XII emission observed by the UVCS slit. At the same time, the curved shape of the white light brightening (see bottom frames of Fig. 2) is similar to that of the opening loop detected earlier by EIT. 

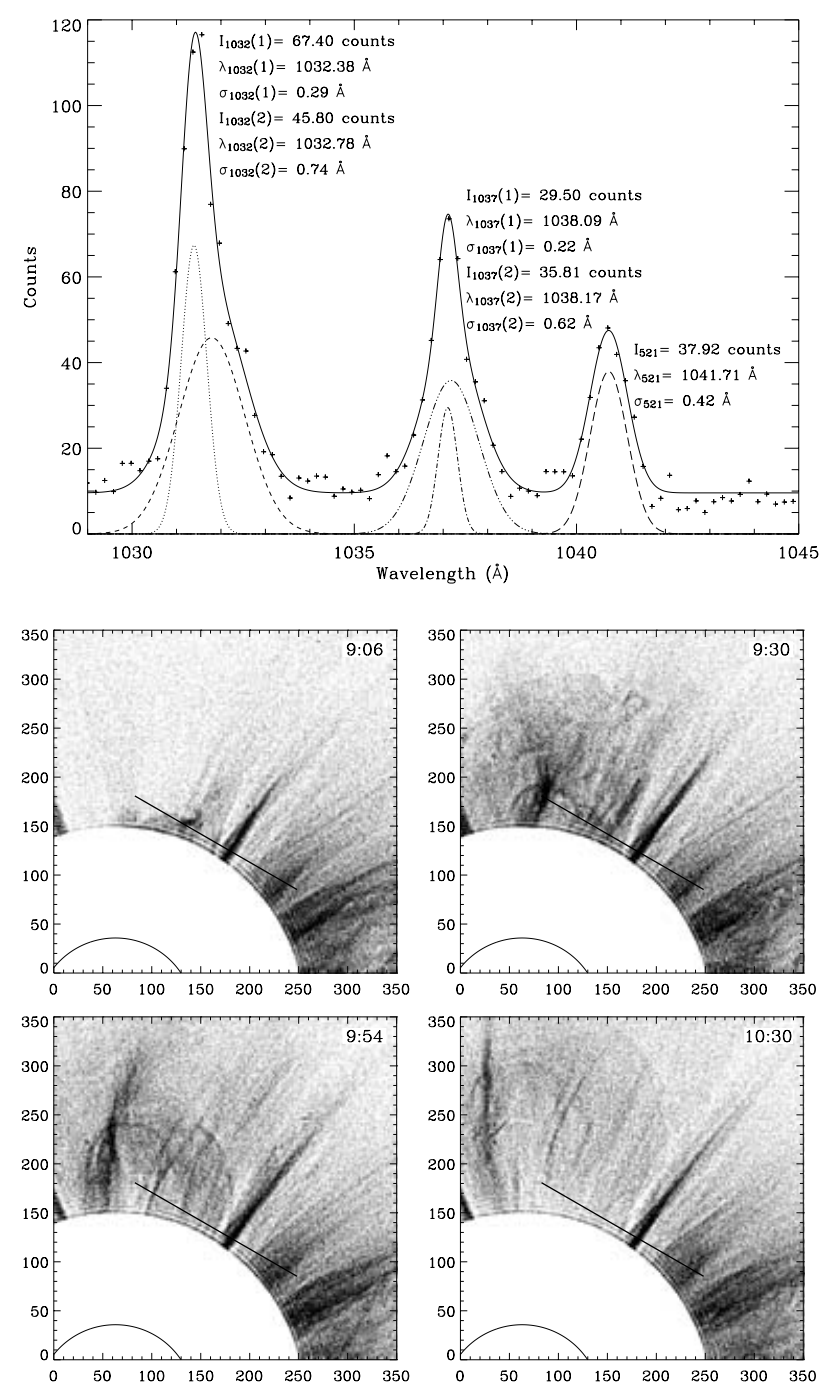
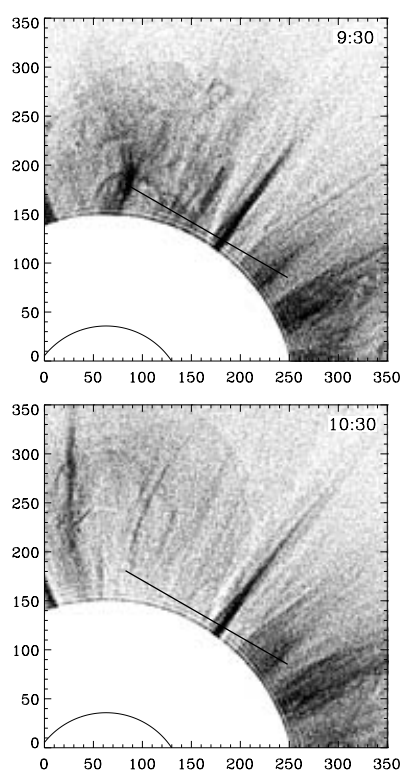

Figure 1. Spectra integrated over the spatial pixels where the hot gas emission (intensity enhancement of the Si XII lines) is detected in the UVCS data (+ signs). The multi-Gaussian fit of the data is also plotted (solid curve) together with the individual Gaussians. The O vi lines are fitted by two Gaussians each and the Si XII line by one. The fit parameters are also given (in counts for the amplitude and in wavelength unit for the center and width of each Gaussian).

\section{Discussion}

Figure 2. LASCO C2 images giving the evolution in space and time of the erupted system of loops as well as the CME material observed in white light. The black straight lines indicate the location of the UVCS slit. The opening system of loops observed in white light is highly correlated to the hot gas emission seen propagating along the slit of UVCS.

The enhanced emission of the Si XII line and the line broadening observed for the O VI doublet indicates the presence of expanding hot material propagating along the UVCS slit. The EUV propagating structure is well correlated spatially and temporally to the evolution of the white-light LASCO observations of the expanding CME (see Fig. 2). The CME speed was high enough at low heights (considering an estimated projected speed of about $1200 \mathrm{~km} \mathrm{~s}^{-1}$ at the slit position) to be able to drive a coronal shock wave and produce local heating of the plasma that could be responsible for the observed enhanced emission and broadening of the EUV lines in higher ionization states. For more details, see Raouafi et al. (2004).

\section{References}

Mancuso, S., Raymond, J. C., Kohl, J., et al. 2002, A\&A,383, 267

Raouafi, N.-E., Mancuso, S., Solanki, S. K., et al. 2004, A\&A, 424, 1039

Raymond, J. C., Thompson, B. J., St. Cyr, O. C., et al. 2000, GRL, 27, 1439 